\title{
INTEGRATING UAV INTO GEOMATICS CURRICULUM
}

\author{
Raid Al-Tahir \\ Department of Geodesy and Geomatics Engineering, University of New Brunswick, \\ Fredericton, New Brunswick, E3B 5A3 Canada. \\ raid.altahir@unb.ca
}

KEY WORDS: Curriculum Development. Unmanned Aerial Vehicles. Photogrammetry. Geomatics.

\begin{abstract}
:
Unmanned aerial vehicles (UAV) have gained tremendous interest as a platform for surveying and mapping over the last few years, and have opened up a new realm of opportunities for surveying, orthophoto production, 3D modelling and feature extraction. UAVs provide a viable and affordable alternative for the airborne and space borne sensors for the medium/large scale mapping. This paper argues that universities should expand their education and training programs to include UAV-based geomatics operations and application development. Based on the author's own experience as well other cases, details are developed and presented in this paper with respect to the likely syllabi and practical assignments. Alternatives for hardware and software support will be briefly discussed.
\end{abstract}

\section{INTRODUCTION}

\subsection{Background}

The use of unmanned aerial vehicles (UAV) in civilian applications has significantly increased over the last few years and most certainly will continue growing. The Association for Unmanned Vehicle Systems International predicts that by 2025 the UAV industry in the USA alone will generate $\$ 82$ billion and will produce more than 100,000 new UAV-related jobs (AUVSI, 2013).

Consequently, the need for experienced sensor operators and pilots will continue to grow. To fill those jobs, a number of universities have launched unmanned aerial systems pilot and research degree programs. More than 20 community colleges and universities in the USA and some 50 colleges worldwide are taking advantage of this emerging job market by creating specific degree and research programs devoted to UAV systems (Drone Training HQ, 2015; Wynbrandt, 2015).

UAV-related programs in universities are often housed within mechanical engineering, aerospace engineering, or robotics departments. These programs fall into the following typical academic levels.

- Certificate level programs in unmanned aircraft systems pilot and operations, as well as technician/maintenance.

- Minor in unmanned an aerial systems that includes an introduction to unmanned systems operations and the aspects of navigation, obstacle and terrain avoidance systems, on-board processing systems, etc.

- Bachelor of Science in unmanned aircraft systems science that prepare the graduates for employment as operators, observers, sensor operators, and operations administrators of unmanned aircraft systems.

- Master of Science and PhD degrees in unmanned and autonomous systems engineering. These specialized degrees in Mechanical and Aerospace Engineering focuses on unmanned systems engineering and program/project management.
In order to have a leading position in the competition, even universities that don't offer UAV-specific classes have at least first-class unmanned aerial vehicle clubs and research groups (Drone Training HQ, 2015).

\subsection{UAVs in Geomatics}

In a similar fashion, UAVs have gained tremendous interest as a platform for imaging and mapping. The fascination of geomatics industry in the unmanned aerial vehicles is in most parts due to their ability to collect site specific images at high spatial resolution, at low-cost, and with convenient deployment. Additionally, the high resolution imagery enables automated and accurate production of detailed elevation data, orthophotos and 3D landscapes that meet both the company and client needs.

There is already an extensive scope for geomatics applications of UAV imagery. Some of the applications put into operation include archaeology, architecture, cultural heritage, large scale mapping, 3D city modeling, change detection in urban and suburban areas, cadastre, agriculture and forestry, natural and man-made hazards, environmental and construction monitoring (Gruen, 2012).

The signs clearly point in the direction that the use of UAVs has been established, generally accepted, and vigorously growing (Lemmens, 2015). Moreover, a group of experts on Global Geospatial Information Management has identified the increased demand for applying high-resolution imagery and the increase use of Unmanned Aerial Vehicles as a tool for rapid geospatial data collection as key emerging trends for the next ten years (UN-GGIM, 2013).

One may argue in support of the above statements that there is an increasing need for accurate, detailed and up-to-date geoinformation. Such information is crucial for providing assessment and solutions to the global issues of population growth and migration, resource management, as well as the various problems and disasters associated with climate change and sea-level rise (Lemmens, 2015). 
The evolving interest in UAV systems is evident by the wide spread of their use as a new surveying technology by private sectors. Additionally, the increasing interest is also reflected by the growing number of dedicated sessions and workshops in professional conferences. Academic and research institutions have also shown an interest in adopting UAVs for mapping, although still limited and mainly at the level of graduate research and development.

\section{OPTIONS FOR CURRICULUM DEVELOPMENT}

It is obvious that soon there will be a demand for experienced operators as the geomatics applications of UAV systems increase. However, the use of UAVs has not yet widely incorporated into geomatics and geomatics engineering programs. One may reason that UAV as a surveying tool is still evolving, and, moreover, being hindered by the unclear aviation regulations.

Nevertheless, this paper argues that universities should prepare for the demand and pursue more progressively incorporating UAVs in their academic offering. Programs need to include more courses, or expand existing ones, to provide the education and training in UAV-based geomatics operations and application development.

This paper proposes three-tier approach to integrating the teaching of UAV-based technology into the undergraduate programs of geomatics and geomatics engineering. The first level is at the individual technical project, the second level is a short session in a course, while the third level is a significant component or a complete course. The three different levels for UAV education differ by the amount of expected dependency on technology support and allocated time. The following sections provide more details regarding these levels based on the author's own experience (Al-Tahir, 2014) and information on courses offered by other institutions.

\subsection{Capstone Project}

An individual student is typically required to produce a project by the end of the program. Such a project may take the form of a project or a report related to technical, research, or design aspects depending on the specific school and department.

A design undertaking will include practical aspects of data acquisition and flight planning. It may also be expanded to include images processing for generating orthophoto, DEM or a digital map. The scope of such project can cover a historic building or monument, a section of the campus or building compound (as shown in Figure 1). The aim is to get the student (or a small group of students) involved in the photogrammetric design and processes using established/commercial software.

Alternatively, the project can motivate the student to understand and apply UAV images in applications other than topographic mapping. Feasible examples include generating image mosaic for land cover mapping and monitoring with respect to environmental or resource management aspects. Other applications include land management and cadastral aspects, natural and man-made hazards, construction monitoring, mining, and others.

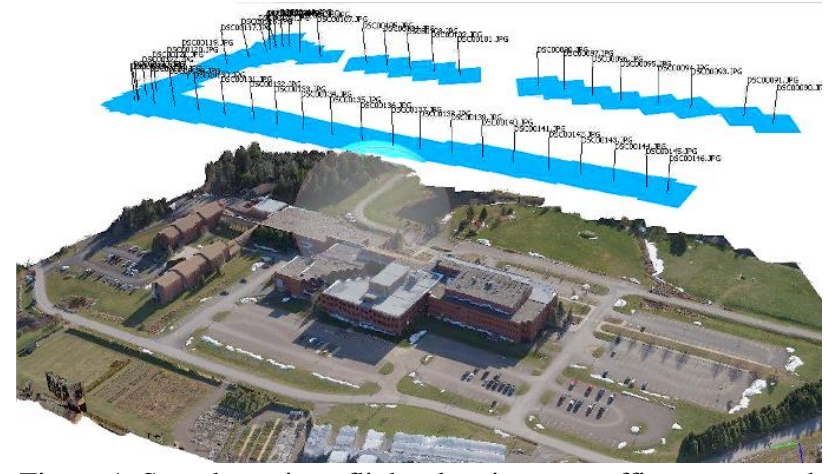

Figure 1. Sample project: flight planning over office compound

The capstone project seems more technically challenging to the student when compared to learning in a class. However, this form is the least demanding in terms of physical resources (class room, laboratory, computers), personnel (instructor, teaching assistant), and time tabling. For that reason, this paper considers this approach as the first tier for integrating UAV in the curriculum of geomatics engineering.

\subsection{Short Module in a Course}

This offering takes the form of few lectures (a session) in an existing photogrammetry course. This session logically should be scheduled for at least the second half of the course after students had already introduced to the basic principles of photogrammetry.

This level of integrating UAV in the curriculum differs from the first one in that all the students in a class (rather than just one or two) are introduced to the UAV as a platform for image collection. Moreover, it is more demanding in terms of preparation and delivery of course material and practical assignments, as well as class and laboratory spaces and scheduling.

2.2.1 Module Objectives. The student will be able to:

- Define unmanned aerial vehicles.

- List and classify different UAV types.

- Evaluate the use of UAVs over traditional techniques.

2.2.2. Module Syllabus. The module covers the following aspects:

- UAV definition and terminology.

- Market and application sectors.

- Advantages and limitations of UAVs in geomatics.

- UAV types (fixed wing and rotary); Launch and recovery systems.

- Regulations; Health and safety considerations.

The advantage of having this module or session is to provide the students with actual hands-on and exposure to the practical aspects of photogrammetry with respect to flight planning, aerial triangulation, image processing and feature extraction.

A series of short assignments, or one term project, can be developed to cover:

- UAV data acquisition.

- Image processing.

- Ortho-rectification

- Data modeling DEM/DTM

- $3 \mathrm{D}$ feature extraction. 
The UAV will be used in this course as the affordable means for photogrammetry mission design and data acquisition. The students, however, will be using selected commercial off-the shelf software tools, and be able to explain the underlying geometric and mathematical principles behind the processes involved.

\subsection{Advanced Analytical Treatment of UAVs}

This form can be a complete course by itself, or a significant component of an advanced course in photogrammetry that deals with analytical and digital photogrammetry.

Besides the platform, the UAV-based system includes the sensors to capture different data as well as the dedicated software to automatically process the raw data. The photogrammetric processing of UAV data and images is typically proceeds through few critical steps;

1. Analyzing all images to identifying matching points.

2. Performing bundle block adjustment using the matching points and other on-board navigation data.

3. Calculating the $3 \mathrm{D}$ coordinates of all matching points (3D point cloud).

4. Interpolating the 3D points and constructing a dense 3D model to obtain a DEM.

5. Generating ortho-image and ortho-mosaic

6. Extracting natural and cartographic features.

These phases significantly impact on the development the objectives and syllabus for this course.

2.3.1 Course Objective. Following (Abdullah, 2015), students will be able to:

- Describe the basic principles of UAS operation requirements.

- Assess available UAV system and data processing software and their suitability for the job.

- Design a UAS-based aerial imagery operation and flight mission.

- Recommend a procedure for sensor calibration.

- Generate digital elevation models, orthophotos, and photo-mosaics.

2.3.2 Course Syllabus. The course assumes that students had a prior knowledge of principles of photogrammetry and remote sensing. Accordingly, possible topics for the course can be (Abdullah, 2015; CATUAV, 2015):

- Review of photogrammetry:

- Photogrammetric principles.

- Sensor calibration.

- Digital elevation model generation.

- Orthophoto mosaic generation.

- UAV technology:

○ Technology review; sensors and platforms.

- Robotics and computer vision.

- Legislation, safety, security and privacy issues.

- Flight planning and operation.

- Hands-on practical training:

- Data processing software.

- Planning and operation of autonomous UAV.

- Image pre-processing and aerial triangulation.

- Generation of orthophoto mosaics and DEM
- Civilian and remote sensing applications:

$\begin{array}{ll}\circ & \text { Earth Observation. } \\ \circ & \text { Precision Agriculture. } \\ \circ & \text { Environmental. } \\ \circ & \text { Mining. }\end{array}$

\section{REQUIRED RESOURCES}

All proposed levels for incorporating the teaching of UAV in undergraduate program in geomatics engineering have similar requirements with respect to the hardware and software. The main difference is the number of hardware units and software licences that must be available at any time. While the capstone project option will be satisfied by one unit, having more than one unit and more than one licence for the software will be a must in order to have a smooth and effective run of the course. Another difference is that the advanced course will require more sophisticated software with a possibility of user development and interoperability with other software.

There is a wide variety and options with respect to choosing a UAV platform, navigation and control subsystems, and sensors. Depending on available fund and specific needs, one may choose to gradually build the UAV system in a modular mode, or purchase a complete turn-key UAV mapping system. Either option, such hardware is continuously evolving and changing. The decision can only be made after an investigation into what is available in terms of platform, sensors, and processing software as well as services at the time. A good starting point is the listing on Geo-matching,com (www.geo-matching.com/ category/id64-uas-for-mapping-and-3d-modelling.html).

With respect to UAV image processing, there are several alternatives for acquiring the needed software. While some commercial software is a platform dependent, others are more general. Agisoft Photoscan (www.agisoft.com) and Pix4DMapper (pix4d.com) are normally mentioned in the general software categories that can automatically process UAV and aerial imagery and produce accurate true orthomosaics, point clouds, and raster DSMs. It is also possible to get a trial for both packages.

Another category of packages for working with UAV and other airborne platform is for those that extract information from motion, such as MotionDSP (www.motiondsp.com) and VisualSFM (ccwu.me/vsfm/).

On the lower end, one may be able to use the free image stitching packages for automatic image registration and mosaicking, such as Regeemy (regeemy.software.informer. com), Image Composite Editor (ICE) (research.microsoft.com/ en-us/um/redmond/groups/ivm/ice/), and the open source alternatives gdal (www.gdal.org/gdal_merge.html).

\section{CONCLUSION}

UAV-based systems have gained interest as a new technology for surveying and mapping. There is already an extensive record for geomatics applications of UAV imagery. Moreover, several indicators point to a vigorously growing demand for using UAVs for rapid geospatial data collection for the next ten years.

Consequently, there will be a demand for experienced operators as the geomatics applications of UAV systems grow. This paper 
recommends that geomatics engineering programs should incorporate UAV systems in their academic offerings. It draws on the experience of several universities worldwide that created UAV-related degree and research programs taking advantage of the mounting need for skilled sensor operators and pilots.

Based on personal involvement and observing the recent efforts by others, this paper proposes three different approaches to implement such integration. Each approach differs from the others by the amount of expected demand for technology and academic support.

The provided details can be considered by others as a model and a starting point for developing their own approach. The propped forms can also serve as models for professional development courses to be offered to practicing surveyors and managers of the environment or natural resources.

Further development to these ideas can proceed along formulating the modules within a blended learning environment. Another tactic is to design a tailored set of webbased lectures on the use UAV in geomatics applications similar to the initiative by the Department of Geography in Gent University, Belgium (Ooms et al., 2015).

\section{REFERENCES}

Abdullah, Q. 2015. Geospatial Applications of Unmanned Aerial Systems (GEOG 597G). College of Earth and Mineral Sciences, Penn State University. www.e-education.psu.edu/ geog $597 \mathrm{~g} /$ node $/ 508$.

Al-Tahir, R. 2014. Training and research for developing applications using UAV Imagery. Presented in National Surveyors Conference 2014, St Andrews, Canada.

AUVSI (Association for Unmanned Vehicle Systems International), 2013. The Economic Impact of Unmanned Aircraft Systems Integration in the United States. 38 pages http://www.auvsi.org/econreport (15 Aug. 2015).

CATUAV Tech Center. 2015. UAV Remote Sensing Applications (5th edition). www.catuav.com/ctc/index.php/uavremote-sensing-principles-5 (15 Aug. 2015).

Drone Training HQ, 2015. Unmanned Aerial Vehicle (UAV) Training and Careers. http://dronetraininghq.com (15 Aug. 2015).

Gruen A., 2012. Unmanned Aerial Vehicles - From Toys to Tools. GEOInformatics, Volum 15, Issue 1, pp 14-16.

Lemmens, M., 2015. Confusion. GIM International. Volume 29, Issue 5, page 13.

Ooms, K., De Maeyer, P., De Wit, B., Maddens, R., Nuttens, T., Van de Weghe, N. and Vervust, S. 2015. Design and use of web lectures to enhance GIS teaching and learning strategies: the students' opinions. Cartography And Geographic Information Science. 42(3). p.271-282.

UN-GGIM. 2013. Future Trends in Geospatial Information Management: the five to ten year vision. United Nations initiative on Global Geospatial information Management. http://ggim.un.org/docs/Future-trends.pdf. (15 Aug. 2015).

Wynbrandt, J., 2015. Growing job demand in unmanned aerial systems. www.flyingmag.com/careers/growing-job-demandunmanned-aerial-systems\#6JJV4MIUxFbEVMyj.99. (15 Aug. 2015). 